Research Article

\title{
VDB Entropy Measures and Irregularity-Based Indices for the Rectangular Kekulene System
}

\author{
Weidong Zhao $\mathbb{D}^{1},{ }^{1}$ K. Julietraja $\mathbb{D}^{2},{ }^{2}$ P. Venugopal $\mathbb{D}^{3},{ }^{3}$ and Xiujun Zhang ${ }^{1}$ \\ ${ }^{1}$ School of Computer Science, Chengdu University, Chengdu, China \\ ${ }^{2}$ Department of Mathematics, Sri Sivasubramaniya Nadar College of Engineering, Kalavakkam 603110, India \\ ${ }^{3}$ Mathematics, School of Science \& Humanities, Shiv Nadar University Chennai, Kalavakkam 603110, India \\ Correspondence should be addressed to K. Julietraja; julietrajak@ssn.edu.in
}

Received 28 August 2021; Accepted 18 November 2021; Published 14 December 2021

Academic Editor: Musavarah Sarwar

Copyright (c) 2021 Weidong Zhao et al. This is an open access article distributed under the Creative Commons Attribution License, which permits unrestricted use, distribution, and reproduction in any medium, provided the original work is properly cited.

Theoretical chemists are fascinated by polycyclic aromatic hydrocarbons (PAHs) because of their unique electromagnetic and other significant properties, such as superaromaticity. The study of PAHs has been steadily increasing because of their wideranging applications in several fields, like steel manufacturing, shale oil extraction, coal gasification, production of coke, tar distillation, and nanosciences. Topological indices (TIs) are numerical quantities that give a mathematical expression for the chemical structures. They are useful and cost-effective tools for predicting the properties of chemical compounds theoretically. Entropic network measures are a type of TIs with a broad array of applications, involving quantitative characterization of molecular structures and the investigation of some specific chemical properties of molecular graphs. Irregularity indices are numerical parameters that quantify the irregularity of a molecular graph and are used to predict some of the chemical properties, including boiling points, resistance, enthalpy of vaporization, entropy, melting points, and toxicity. This study aims to determine analytical expressions for the VDB entropy and irregularity-based indices in the rectangular Kekulene system.

\section{Introduction}

Chemical graph theory is one of the important branches of mathematics, which helps researchers to explore chemical compounds by reconstructing their underlying molecular structures as graphs. A molecular graph is a simple graph that represents the skeleton of an organic chemical compound in which the vertices of the molecular graph represent carbon atoms, and its edges represent the links between those carbon atoms [1]. According to IUPAC, the molecular structural descriptor is described as "A molecular structural descriptor is a numerical quantity associated with the chemical constitution that can be used to correlate chemical structure with several physical characteristics, biological activity, or chemical reactivity" [2].

Cycloarenes are conjugated macrocyclic chemical compounds composed of circularly fused benzene rings that enclose a cavity with inward-facing $\mathrm{C}-\mathrm{H}$ bonds. Thus, cycloarenes are a subdivision of the Kekulene system. The magnetic susceptibility, aromaticity, and vibrational frequencies of cycloarenes have all been the subject of theoretical research $[3,4]$.

The rectangular Kekulene system $\operatorname{RK}(m, n)$ is a type of polycyclic aromatic compound that has a broad range of applications, from everyday materials to cutting-edge industries like nanotechnology. As a result, theoretical chemists have long been attracted to the study of their fundamental features, including carcinogenicity, toxicity, observed bioactivities, and other relevant features. The fascinating potential of increased stability with interesting magnetic and magnetocaloric effects is offered by the structure of the Kekulene molecule, which has 12 annulated benzene rings and a central cavity.

Numerous theoretical and experimental studies on coronoids have been sparked by the synthesis of Kekulene and septulene [5-7]. Due to their intriguing property of superaromaticity, the study of Kekulene systems is also gaining momentum. Extra thermodynamic stability owing 
to macrocyclic conjugation in super-ring compounds like Kekulene is referred to as superaromaticity or macrocyclic aromaticity, and it accounts for a minor portion of global aromaticity. All of the coronoids that have been investigated so far are superaromatic in some way, with positive superaromatic stabilisation energies (SSEs). This gives rise to a more in-depth investigation of the features of Kekulene systems and their linkages to the underlying molecular structures. This research can be used in a variety of nanotechnology fields.

The cavities, which are an intrinsic element of the Kekulene system, serve as prototypes for developing and synthesizing novel nanomaterials with applications in nanotechnology and biotechnology, as well as the burgeoning field of nanomedicine. They have also been used in the design and synthesis of porous and mesoporous materials based on calixarenes and mesoporous silica for the storage and complex formation of hazardous nuclear waste and other contaminants [8-10]. It has been demonstrated that by utilizing Kekulene system knowledge, one can accurately compute total pi-electron energy, resonance energy, and coronoid hydrocarbon enumeration [11].

Graph-theoretical techniques are more efficient in acquiring the properties of the Kekulene system than computationally intensive quantum chemical calculations. Aihara [12] pointed out in a recent study that graph theory is beneficial not only for predicting topological resonance energies but also for exposing severe flaws in prior aromaticity theories. Recently, Julietraja and Venugopal computed the degree-based indices for coronoid structures [13]. Julietraja et al. analyzed the degree-based molecular descriptors using M-polynomial for certain classes of benzenoid systems $[14,15]$. Julietraja et al. used entropy measures to investigate three prominent classes of PAHs [16].

However, despite the several promising applications of the rectangular kekulene systems, there have been no attempts till now to study these systems from a structural perspective. Our research, detailed in this article, is an effort towards bridging this research gap. The objective of this article is to compute the VDB entropy measures and irregularity-based indices for the rectangular Kekulene system. Since we tackle two open problems in this article, the number of known TIs for these systems will be doubled. This increased number of known molecular descriptors will contribute to the current body of knowledge on these systems. The TIs will also form the basis on which new Kekulene structures can be designed and synthesised.

The subsequent sections cover a literature survey on graph-theoretical concepts, degree-based entropy measures, and irregularity-based indices. There is a brief summary of the methods used for research, followed by the computation of the analytical expressions of both entropy measures and irregularity indices for rectangular Kekulene systems. The analytical expressions of the descriptors are used to compute the numerical values and observe their behaviour using graphical plots.

\section{Graph-Theoretical Concepts}

In this article, $\Gamma_{1}(V, E)$ denotes a connected graph, where $V$ and $E$ denote the vertex and edge sets, respectively. The degree of a vertex $m_{1}$ in a graph $\Gamma_{1}$ is the number of edges that are adjacent to that vertex $m_{1}$ and is denoted by $\operatorname{deg}_{\Gamma_{1}}\left(m_{1}\right)$ [17]. There is a large body of knowledge about degree-based topological descriptors, which have a strong co-relation with numerous physicochemical properties of PAHs such as Zagreb and its co-indices, ABC index, GA index, Randić index, sum-connectivity index, and SDD index. These indices are also used to generate degree-based entropy measures.

2.1. Edge Weight- and Degree-Based Entropy. Let $n$ be the order of a graph of size $m$ and $\varphi$ be some meaningful information function. The Shannon's entropy $[18,19]$ of a graph $\Gamma_{1}$ is defined as

$$
\operatorname{ENT}_{\varphi}\left(\Gamma_{1}\right)=-\sum_{l=1}^{n} \frac{\varphi\left(\operatorname{deg}_{\Gamma_{1}}\left(v_{l}\right)\right)}{\sum_{m=1}^{n} \varphi\left(\operatorname{deg}_{\Gamma_{1}}\left(v_{m}\right)\right)} \log \left[\frac{\varphi\left(\operatorname{deg}_{\Gamma_{1}}\left(v_{l}\right)\right)}{\sum_{m=1}^{n} \varphi\left(\operatorname{deg}_{\Gamma_{1}}\left(v_{m}\right)\right)}\right]
$$

Let $v_{l} \in V\left(\Gamma_{1}\right)$ and the degree of $v_{l}$ be represented by the information function $\varphi\left(v_{l}\right)$, that is, $\varphi\left(v_{l}\right)=\operatorname{deg}_{\Gamma_{1}}\left(v_{l}\right)$. Then, equation (1) can be rewritten as

$$
\operatorname{ENT}_{\varphi}\left(\Gamma_{1}\right)=-\sum_{l=1}^{n} \frac{\operatorname{deg}_{\Gamma_{1}}\left(v_{l}\right)}{\sum_{m=1}^{n} \operatorname{deg}_{\Gamma_{1}}\left(v_{m}\right)} \log \left[\frac{\operatorname{deg}_{\Gamma_{1}}\left(v_{l}\right)}{\sum_{m=1}^{n} \operatorname{deg}_{\Gamma_{1}}\left(v_{m}\right)}\right] .
$$

According to the fundamental theorem of graph theory, $\sum_{m=1}^{n} \operatorname{deg}_{\Gamma_{1}}\left(v_{m}\right)=2 E$. Hence, equation (2) reduces to

$$
\operatorname{ENT}_{\varphi}\left(\Gamma_{1}\right)=\log (2 E)-\frac{1}{2 E} \log \left[\sum_{l=1}^{n}\left(\operatorname{deg}_{\Gamma_{1}}\left(v_{l}\right)\right)^{\operatorname{deg}_{\Gamma}\left(v_{l}\right)}\right] .
$$

The entropy measure of an edge-weighted graph was first proposed by Chen et al. [20]. If $\Gamma_{1}=\left(V\left(\Gamma_{1}\right) ; E\left(\Gamma_{1}\right) ; \varphi\left(l_{1} m_{1}\right)\right.$ is an edge-weighted graph, where $V\left(\Gamma_{1}\right), E\left(\Gamma_{1}\right)$, and $\varphi\left(l_{1} m_{1}\right)$ represents vertex set, edge set, and edge weight of the edge $\left(l_{1} m_{1}\right)$ of $\Gamma_{1}$, then we have 


$$
\operatorname{ENT}_{\varphi}\left(\Gamma_{1}\right)=-\sum_{l_{1}^{\prime} m_{1}^{\prime} \in E(\Gamma)} \frac{\varphi\left(l_{1}^{\prime} m_{1}^{\prime}\right)}{\sum_{l_{1} m_{1} \in E\left(\Gamma_{1}\right)} \varphi\left(l_{1} m_{1}\right)} \log \left[\frac{\varphi\left(l_{1}^{\prime} m_{1}^{\prime}\right)}{\sum_{l_{1} m_{1} \in E\left(\Gamma_{1}\right)} \varphi\left(l_{1} m_{1}\right)}\right] .
$$

By using the equations of (1), (3), and (4), we get the VDB entropy measures listed in Table 1.

2.2. Irregularity-Based Indices for $Q S P R$ Analysis. Irregularity indices are numerical parameters that quantify the irregularity of a molecular graph. The study of irregular graphs was brought to the limelight by Paul Erdös in [21]. Erdös raised an open question on irregular graphs [22], at the Second Krakow Conference on Graph Theory.

If a topological descriptor becomes zero for a normal graph and nonzero for a nonregular graph, it is known as an irregularity index. Bell proposed the first irregularity index in [23]. Irregularity indices have proved to be particularly useful in quantifying the topology of nonregular graphs. In QSPR and QSAR studies, irregularity of graphs is used to predict a range of physical and chemical properties, including melting and boiling points, enthalpy of vaporization, resistance, entropy, and toxicity. It has been proved that using regression analysis in [24], 4-octane isomer properties such as enthalpy of vaporization (HVAP), entropy, acentric factor (AcenFac), and standard enthalpy of vaporization (DHVAP) can be determined using irregularity indices with a correlation coefficient of magnitude higher than 0.9 . Some commonly used irregularity indices and their analytical expressions are listed in Table 2.

The recent work and development of irregularity indices can be seen more elaborately in [25-29].

\section{Methods}

In this paper, the computations are performed using graphtheoretical methods, the edge partition method, and analytical techniques. Maple is utilized in obtaining the analytical expressions for the degree-based entropy measures. Maple is used to calculate the numerical values of the VDB entropy measures and irregularity indices based on analytical expressions. The numerical results are represented visually using Origin, and Chem Draw Ultra is used for describing the molecular structures of the three PAHs.

3.1. Degree-Based Entropy for the Rectangular Kekulene System. Let $\Gamma$ be a rectangular Kekulene system $\operatorname{RK}(r, s)$. The number of vertices and edges of $\operatorname{RK}(r, s)$ is $V\left(\Gamma_{1}\right)=$ $36 r s+14 s-2 r \quad$ and $\quad E\left(\Gamma_{1}\right)=48 r s+16 s-4 r . \quad \operatorname{RK}(r, s)$ consists of $12 r s+10 s+2 r$ vertices of degree 2 and $24 r s+$ $4 s-4 r$ vertices of degree 3 . It is picturised in Figure 1. The edge partition table of rectangular Kekulene system $\operatorname{RK}(r, s)$ is represented in Table 3.

3.2. First Zagreb Entropy. By using the edge partition in Table 3,

$$
M_{1}\left(\Gamma_{1}\right)=(264 s-28) r+76 s .
$$

Then, using the equation of first Zagreb entropy, the result is obtained as

$$
\begin{aligned}
\operatorname{ENT}_{M_{1}}\left(\Gamma_{1}\right)= & \log \left(M_{1}\left(\Gamma_{1}\right)\right)-\frac{1}{M_{1}\left(\Gamma_{1}\right)} \log \left[\prod_{l_{1} m_{1} \in E\left(\Gamma_{1}\right)}\left[\operatorname{deg}_{\Gamma_{1}}\left(l_{1}\right)+\operatorname{deg}_{\Gamma_{1}}\left(m_{1}\right)\right]^{\left[\operatorname{deg}_{\Gamma_{1}}\left(l_{1}\right)+\operatorname{deg}_{\Gamma_{1}}\left(m_{1}\right)\right]}\right] \\
= & {\left[\prod_{l_{1} m_{1} \in E_{\{2,2\}}}\left[\operatorname{deg}_{\Gamma_{1}}\left(l_{1}\right)+\operatorname{deg}_{\Gamma_{1}}\left(m_{1}\right)\right]^{\left[\operatorname{deg}_{\Gamma_{1}}\left(l_{1}\right)+\operatorname{deg}_{\Gamma_{1}}\left(m_{1}\right)\right]}\right] } \\
& \log \left(M_{1}(\Gamma)\right)-\frac{1}{M_{1}(\Gamma)} \log \left[\prod_{l_{1} m_{1} \in E_{\{2,3\}}}\left[\operatorname{deg}_{\Gamma_{1}}\left(l_{1}\right)+\operatorname{deg}_{\Gamma_{1}}\left(m_{1}\right)\right]^{\left[\operatorname{deg}_{\Gamma_{1}}\left(l_{1}\right)+\operatorname{deg}_{\Gamma_{1}}\left(m_{1}\right)\right]}\right] \\
= & \left.\log ((264 s-28) r+76 s)-\frac{1}{[(264 s-28) r+76 s]}\right] \\
& \log \left[\left[[4 s+2 r] \times[4]^{[4]}\right] \times\left[[24 r s+12 s] \times[5]^{[5]}\right] \times\left[[24 r s-6 r] \times[6]^{[6]}\right]\right] .
\end{aligned}
$$




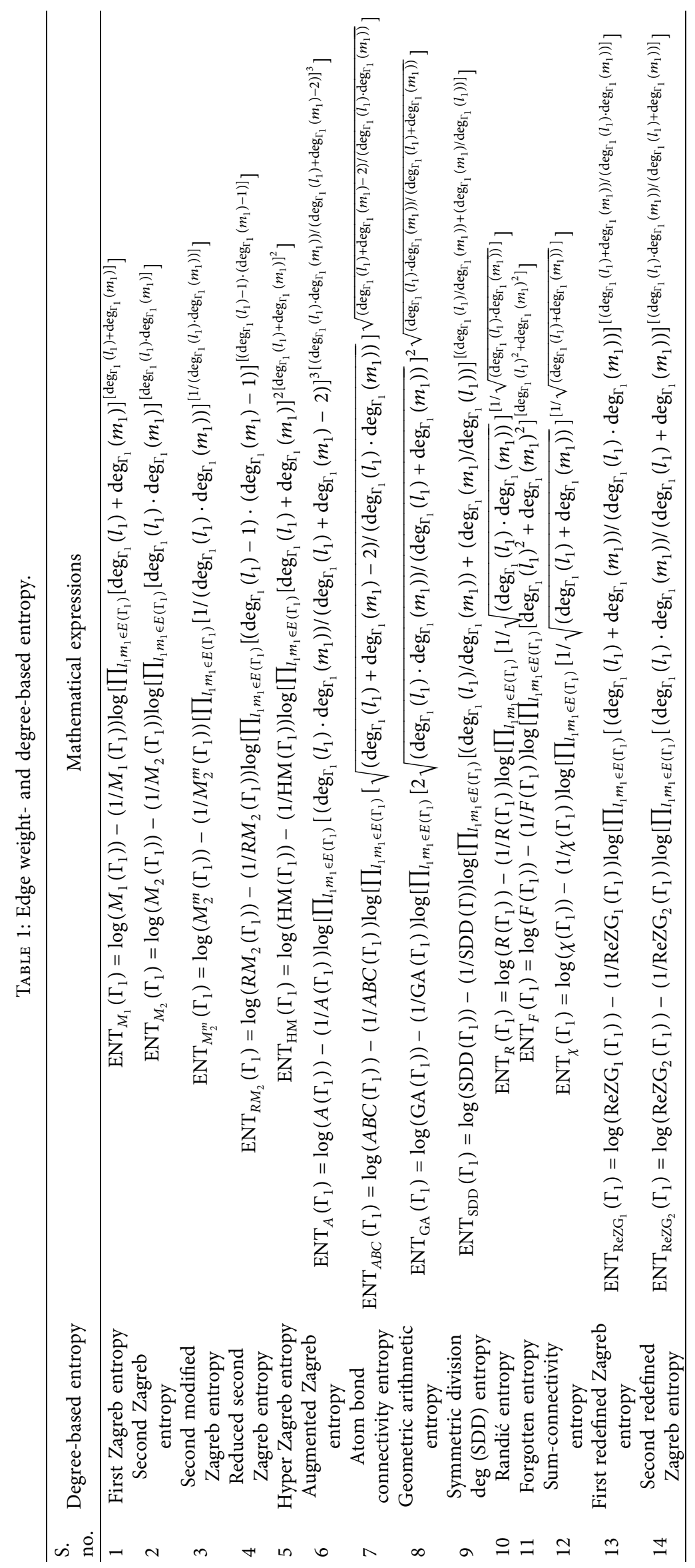


TABLE 2: Irregularity-based indices for QSPR analysis.

\begin{tabular}{|c|c|}
\hline S. no. & Irregularity-based indices \\
\hline 1 & $\operatorname{VAR}\left(\Gamma_{1}\right)=\sum_{l_{1} \in V\left(\Gamma_{1}\right)}\left(\operatorname{deg}_{\Gamma_{1}}\left(l_{1}\right)-(2 m / n)\right)^{2}=\left(M_{1}(\Gamma) / n\right)-(2 m / n)^{2}$ \\
\hline 2 & $\operatorname{IR} 1\left(\Gamma_{1}\right)=\sum_{l_{1} \in V\left(\Gamma_{1}\right)} \operatorname{deg}_{\Gamma_{1}}\left(l_{1}\right)^{3}-(2 m / n) \sum_{l_{1} \in V\left(\Gamma_{1}\right)} \operatorname{deg}_{\Gamma_{1}}\left(l_{1}\right)^{2}=F(\Gamma)-(2 m / n) M_{1}(\Gamma)$ \\
\hline 3 & $\operatorname{IR2}\left(\Gamma_{1}\right)=\sqrt{\sum_{l_{1} m_{1} \in E\left(\Gamma_{1}\right)}\left[\operatorname{deg}_{\Gamma_{1}}\left(l_{1}\right) \cdot \operatorname{deg}_{\Gamma_{1}}\left(m_{1}\right)\right] / m}-(2 m / n)=\sqrt{M_{2}(\Gamma) / m}-(2 m / n)$ \\
\hline 4 & $\left.\operatorname{IRDIF}\left(\Gamma_{1}\right)=\sum_{l_{1} m_{1} \in E\left(\Gamma_{1}\right)}\right)\left(\operatorname{deg}_{\Gamma_{1}}\left(l_{1}\right) / \operatorname{deg}_{\Gamma_{1}}\left(m_{1}\right)\right)-\left(\operatorname{deg}_{\Gamma_{1}}\left(m_{1}\right) / \operatorname{deg}_{\Gamma_{1}}\left(m_{1}\right)\right) \mid$ \\
\hline 5 & $\operatorname{AL}\left(\Gamma_{1}\right)=\sum_{l_{1} m_{1} \in E\left(\Gamma_{1}\right)}\left|\operatorname{deg}_{\Gamma_{1}}\left(l_{1}\right)-\operatorname{deg}_{\Gamma_{1}}\left(m_{1}\right)\right|$ \\
\hline 6 & $\operatorname{IRL}\left(\Gamma_{1}\right)=\sum_{l_{1} m_{1} \in E\left(\Gamma_{1}\right)} \ln \operatorname{deg}_{\Gamma_{1}}\left(l_{1}\right)-\ln \operatorname{deg}_{\Gamma_{1}}\left(m_{1}\right) \mid$ \\
\hline 7 & $\operatorname{IRLU}\left(\Gamma_{1}\right)=\sum_{l_{1} m_{1} \in E\left(\Gamma_{1}\right)}\left(\left|\operatorname{deg}_{\Gamma_{1}}\left(l_{1}\right)-\operatorname{deg}_{\Gamma_{1}}\left(m_{1}\right)\right| / \min \left(\operatorname{deg}_{\Gamma_{1}}\left(l_{1}\right) \cdot \operatorname{deg}_{\Gamma_{1}}\left(m_{1}\right)\right)\right)$ \\
\hline 8 & $\operatorname{IRLF}\left(\Gamma_{1}\right)=\sum_{l_{1} m_{1} \in E\left(\Gamma_{1}\right)}\left(\left|\operatorname{deg}_{\Gamma_{1}}\left(l_{1}\right)-\operatorname{deg}_{\Gamma_{1}}\left(m_{1}\right)\right| / \sqrt{\left(\operatorname{deg}_{\Gamma_{1}}\left(l_{1}\right) \cdot \operatorname{deg}_{\Gamma_{1}}\left(m_{1}\right)\right)}\right)$ \\
\hline 9 & $\operatorname{IRF}\left(\Gamma_{1}\right)=\sum_{l_{1} m_{1} \in E\left(\Gamma_{1}\right)}\left(d_{u}-d_{v}\right)^{2}=F\left(\Gamma_{1}\right)-2 M_{2}\left(\Gamma_{1}\right)$ \\
\hline 10 & $\operatorname{IRLA}\left(\Gamma_{1}\right)=\sum_{l_{1} m_{1} \in E\left(\Gamma_{1}\right)}\left(\left|\operatorname{deg}_{\Gamma_{1}}\left(l_{1}\right)-\operatorname{deg}_{\Gamma_{1}}\left(m_{1}\right)\right| /\left(\operatorname{deg}_{\Gamma_{1}}\left(l_{1}\right)+\operatorname{deg}_{\Gamma_{1}}\left(m_{1}\right)\right)\right)$ \\
\hline 11 & $\operatorname{IRD}_{1}\left(\Gamma_{1}\right)=\sum_{l_{1} m_{1} \in E\left(\Gamma_{1}\right)} \ln \left\{1+\left|\operatorname{deg}_{\Gamma_{1}}\left(l_{1}\right)-\operatorname{deg}_{\Gamma_{1}}\left(m_{1}\right)\right|\right\}$ \\
\hline 12 & $\operatorname{IRA}\left(\Gamma_{1}\right)=\sum_{l_{1} m_{1} \in E\left(\Gamma_{1}\right)}\left(\operatorname{deg}_{\Gamma_{1}}\left(l_{1}\right)^{-1 / 2}-\operatorname{deg}_{\Gamma_{1}}\left(m_{1}\right)^{-1 / 2}\right)^{2}$ \\
\hline 13 & $\operatorname{IRB}\left(\Gamma_{1}\right)=\sum_{l_{1} m_{1} \in E\left(\Gamma_{1}\right)}\left(\operatorname{deg}_{\Gamma_{1}}\left(l_{1}\right)^{-1 / 2}-\operatorname{deg}_{\Gamma_{1}}\left(m_{1}\right)^{-1 / 2}\right)^{2}$ \\
\hline 14 & $\operatorname{IRB}\left(\Gamma_{1}\right)=\left(\sum_{l_{1} m_{1} \in E\left(\Gamma_{1}\right)} \sqrt{\left(\operatorname{deg}_{\Gamma_{1}}\left(l_{1}\right) \cdot \operatorname{deg}_{\Gamma_{1}}\left(m_{1}\right)\right)} / m\right)-(2 m / n)=\left(\operatorname{RR}\left(\Gamma_{1}\right) / m\right)-(2 m / n)$ \\
\hline 15 & $\operatorname{IRGA}\left(\Gamma_{1}\right)=\sum_{l_{1} m_{1} \in E\left(\Gamma_{1}\right)} \ln \left(\operatorname{deg}_{\Gamma_{1}}\left(l_{1}\right)+\operatorname{deg}_{\Gamma_{1}}\left(m_{1}\right) / 2 \sqrt{\left(\operatorname{deg}_{\Gamma_{1}}\left(l_{1}\right) \cdot \operatorname{deg}_{\Gamma_{1}}\left(m_{1}\right)\right)}\right)$ \\
\hline 16 & $\operatorname{IRR}_{t}\left(\Gamma_{1}\right)=(1 / 2) \sum_{l_{1} m_{1} \in E\left(\Gamma_{1}\right)}\left|\operatorname{deg}_{\Gamma_{1}}\left(l_{1}\right)-\operatorname{deg}_{\Gamma_{1}}\left(m_{1}\right)\right|$ \\
\hline
\end{tabular}

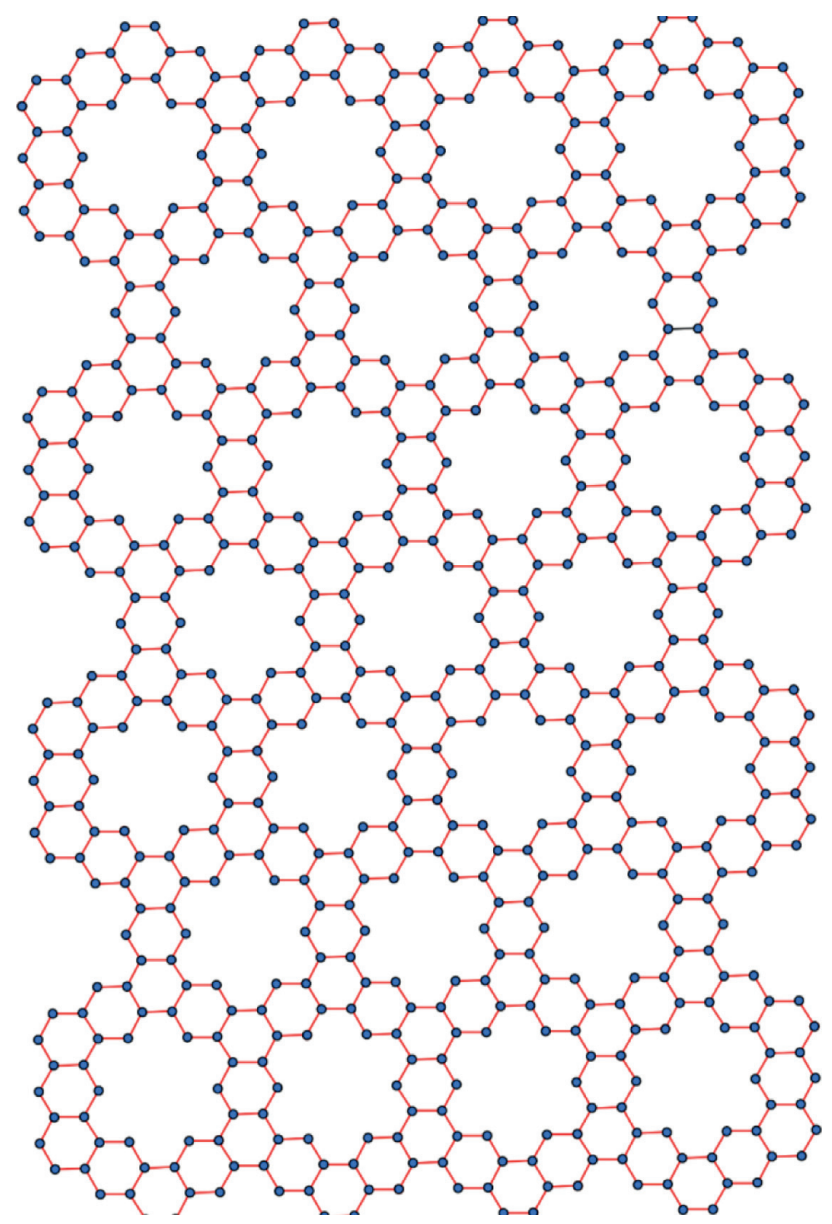

Figure 1: Rectangular Kekulene system. 
TABLE 3: Edge partition table of $\operatorname{RK}(r, s)$.

\begin{tabular}{lc}
\hline$\left(\operatorname{deg}_{\Gamma_{1}}\left(l_{1}\right), \operatorname{deg}_{\Gamma_{1}}\left(m_{1}\right)\right)$ & Total number of edges \\
\hline$(2,2)$ & $4 s+2 r$ \\
$(2,3)$ & $24 r s+12 s$ \\
$(3,3)$ & $24 r s-6 r$ \\
\hline
\end{tabular}

$$
M_{2}\left(\Gamma_{1}\right)=(360 s-46) r+88 s
$$

Then, using the equation of second Zagreb entropy, the result is obtained as

3.3. Second Zagreb Entropy. By using the edge partition in Table 3,

$$
\begin{aligned}
& \operatorname{ENT}_{M_{2}}\left(\Gamma_{1}\right)= \log \left(M_{2}\left(\Gamma_{1}\right)\right)-\frac{1}{M_{2}\left(\Gamma_{1}\right)} \log \left[\prod_{l_{1} m_{1} \in E\left(\Gamma_{1}\right)}\left[\operatorname{deg}_{\Gamma_{1}}\left(l_{1}\right) \cdot \operatorname{deg}_{\Gamma_{1}}\left(m_{1}\right)\right]\right. \\
& {\left[\prod_{l_{1} m_{1} \in E_{\{2,2\}}}\left[\operatorname{deg}_{\Gamma_{1}}\left(l_{1}\right) \cdot \operatorname{deg}_{\Gamma_{1}}\left(m_{1}\right)\right]\right.} \\
&=\left.\log \left(M_{2}\left(\Gamma_{1}\right)\right)-\frac{1}{M_{2}\left(\Gamma_{1}\right)} \log \left[\prod_{l_{1} m_{1} \in E_{\{2,3\}}}\left[\operatorname{deg}_{\Gamma_{1}}\left(l_{1}\right) \cdot \operatorname{deg}_{\Gamma_{1}}\left(m_{1}\right)\right]^{\left[\operatorname{deg}_{\Gamma_{1}}\left(l_{1}\right) \cdot \operatorname{deg}_{\Gamma_{1}}\left(m_{1}\right)\right]}\left(m_{1}\right)\right]^{\left[\operatorname{deg}_{\Gamma_{1}}\left(l_{1}\right) \cdot \operatorname{deg}_{\Gamma_{1}}\left(m_{1}\right)\right]}\right] \\
&\left.\times \prod_{l_{1} m_{1} \in E_{\{3,3\}}}\left[\operatorname{deg}_{\Gamma_{1}}\left(l_{1}\right) \cdot \operatorname{deg}_{\Gamma_{1}}\left(m_{1}\right)\right]^{\left[\operatorname{deg}_{\Gamma_{1}}\left(l_{1}\right) \cdot \operatorname{deg}_{\Gamma_{1}}\left(m_{1}\right)\right]}\right] \\
&= \log ((360 s-46) r+88 s)-\frac{1}{[(360 s-46) r+88 s]} \\
& \log \left[\left[[4 s+2 r] \times[4]^{[4]}\right] \times\left[[24 r s+12 s] \times[6]^{[6]}\right] \times\left[[24 r s-6 r] \times[9]{ }^{[9]}\right]\right] .
\end{aligned}
$$

3.4. Second Modified Zagreb Entropy. By using the edge partition in Table 3,

$$
M_{2}^{m}(\Gamma)=\frac{1}{6}(40 s-1) r+3 s .
$$

Then, using the equation of the second modified Zagreb entropy, the result is obtained as

$$
\begin{aligned}
& \operatorname{ENT}_{M_{2}^{m}}\left(\Gamma_{1}\right)=\log \left(M_{2}^{m}\left(\Gamma_{1}\right)\right)-\frac{1}{M_{2}^{m}\left(\Gamma_{1}\right)} \log \left[\prod_{l_{1} m_{1} \in E\left(\Gamma_{1}\right)}\left[\frac{1}{\left(\operatorname{deg}_{\Gamma_{1}}\left(l_{1}\right) \cdot \operatorname{deg}_{\Gamma_{1}}\left(m_{1}\right)\right)}\right]^{\left[1 /\left(\operatorname{deg}_{\Gamma_{1}}\left(l_{1}\right) \cdot \operatorname{deg}_{\Gamma_{1}}\left(m_{1}\right)\right)\right]}\right] \\
& =\log \left(M_{2}^{m}\left(\Gamma_{1}\right)\right)-\frac{1}{M_{2}^{m}\left(\Gamma_{1}\right)} \log \left[\begin{array}{l}
\left.\times \prod_{l_{1} m_{1} \in E_{\{2,2\}}}\left[\frac{1}{\left(\operatorname{deg}_{\Gamma_{1}}\left(l_{1}\right) \cdot \operatorname{deg}_{\Gamma_{1}}\left(m_{1}\right)\right)}\right]^{\left[1 /\left(\operatorname{deg}_{\Gamma_{1}}\left(l_{1}\right) \cdot \operatorname{deg}_{\Gamma_{1}}\left(m_{1}\right)\right)\right]}\right] \\
\left.\times \prod_{l_{1} m_{1} \in E_{\{2,3\}}}\left[\frac{1}{\left(\operatorname{deg}_{\Gamma_{1}}\left(l_{1}\right) \cdot \operatorname{deg}_{\Gamma_{1}}\left(m_{1}\right)\right)}\right]^{\left[1 /\left(\operatorname{deg}_{\Gamma_{1}}\left(l_{1}\right) \cdot \operatorname{deg}_{\Gamma_{1}}\left(m_{1}\right)\right)\right]}\right] \\
\left.\times \prod_{l_{1} m_{1} \in E_{\{3,3\}}}\left[\frac{1}{\left(\operatorname{deg}_{\Gamma_{1}}\left(l_{1}\right) \cdot \operatorname{deg}_{\Gamma_{1}}\left(m_{1}\right)\right)}\right]^{\left[1 /\left(\operatorname{deg}_{\Gamma_{1}}\left(l_{1}\right) \cdot \operatorname{deg}_{\Gamma_{1}}\left(m_{1}\right)\right)\right]}\right]
\end{array}\right. \\
& =\log \left(\frac{1}{6}(40 s-1) r+3 s\right)-\frac{1}{[(1 / 6)(40 s-1) r+3 s]}
\end{aligned}
$$




$$
\log \left[\left[[4 s+2 r] \times\left[\frac{1}{4}\right]^{[1 / 4]}\right] \times\left[[24 r s+12 s] \times\left[\frac{1}{6}\right]^{[1 / 6]}\right] \times\left[[24 r s-6 r] \times\left[\frac{1}{9}\right]^{[1 / 9]}\right]\right]
$$

3.5. Reduced Second Zagreb Entropy. By using the edge partition in Table 3,

$$
R M_{2}(\Gamma)=((144 s-22) r+28 s .
$$

$$
\begin{aligned}
& \operatorname{ENT}_{R M_{2}}\left(\Gamma_{1}\right)=\log \left(\operatorname{RM}_{2}\left(\Gamma_{1}\right)\right)-\frac{1}{\operatorname{RM}_{2}\left(\Gamma_{1}\right)} \log \left[\prod_{l_{1} m_{1} \in E\left(\Gamma_{1}\right)}\left[\left(\operatorname{deg}_{\Gamma_{1}}\left(l_{1}\right)-1\right) \cdot\left(\operatorname{deg}_{\Gamma_{1}}\left(m_{1}\right)-1\right)\right]\left[\left(\operatorname{deg}_{\Gamma_{1}}\left(l_{1}\right)-1\right) \cdot\left(\operatorname{deg}_{\Gamma_{1}}\left(m_{1}\right)-1\right)\right]\right] \\
& =\log \left(M_{2}^{m}\left(\Gamma_{1}\right)\right)-\frac{1}{M_{2}^{m}\left(\Gamma_{1}\right)} \log \left[\begin{array}{l}
\prod_{l_{1} m_{1} \in E_{\{2,2\}}}\left[\left(\operatorname{deg}_{\Gamma_{1}}\left(l_{1}\right)-1\right) \cdot\left(\operatorname{deg}_{\Gamma_{1}}\left(m_{1}\right)-1\right)\right]{ }^{\left[\left(\operatorname{deg}_{\Gamma_{1}}\left(l_{1}\right)-1\right) \cdot\left(\operatorname{deg}_{\Gamma_{1}}\left(m_{1}\right)-1\right)\right]} \\
\times \prod_{l_{1} m_{1} \in E_{\{2,3\}}}\left[\left(\operatorname{deg}_{\Gamma_{1}}\left(l_{1}\right)-1\right) \cdot\left(\operatorname{deg}_{\Gamma_{1}}\left(m_{1}\right)-1\right)\right]\left[\left(\operatorname{deg}_{\Gamma_{1}}\left(l_{1}\right)-1\right) \cdot\left(\operatorname{deg}_{\Gamma_{1}}\left(m_{1}\right)-1\right)\right] \\
\left.\times \prod_{l_{1} m_{1} \in E_{\{3,3\}}}\left[\left(\operatorname{deg}_{\Gamma_{1}}\left(l_{1}\right)-1\right) \cdot\left(\operatorname{deg}_{\Gamma_{1}}\left(m_{1}\right)-1\right)\right]^{\left[\left(\operatorname{deg}_{\Gamma_{1}}\left(l_{1}\right)-1\right) \cdot\left(\operatorname{deg}_{\Gamma_{1}}\left(m_{1}\right)-1\right)\right]}\right]
\end{array}\right. \\
& =\log ((144 s-22) r+28)-\frac{1}{[(144 s-22) r+28]} \\
& \log \left[\left[[4 s+2 r] \times[1]^{[1]}\right] \times\left[[24 r s+12 s] \times[2]^{[2]}\right] \times\left[[24 r s-6 r] \times[4]^{[4]}\right]\right] \text {. }
\end{aligned}
$$

3.6. Hyper Zagreb Entropy. By using the edge partition in Table 3,

$$
\operatorname{HM}\left(\Gamma_{1}\right)=(1464 s-184) r+364 s .
$$

Then, using the equation of reduced second Zagreb entropy, the result is obtained as 


$$
\begin{aligned}
= & \log ((1464 s-184) r+364 s)-\frac{1}{[(1464 s-184) r+364 s]} \\
& \log \left[\left[[4 s+2 r] \times[16]^{[16]}\right] \times\left[[24 r s+12 s] \times[25]^{[25]}\right] \times\left[[24 r s-6 r] \times[36]^{[36]}\right]\right] .
\end{aligned}
$$

3.7. Augmented Zagreb Entropy. By using the edge partition in Table 3,

$$
A\left(\Gamma_{1}\right)=\frac{1}{32}(14892 s-1675) r+128 s .
$$

$$
\begin{aligned}
& \operatorname{ENT}_{A}\left(\Gamma_{1}\right)=\log \left(A\left(\Gamma_{1}\right)\right)-\frac{1}{A\left(\Gamma_{1}\right)} \log \left[\prod_{l_{1} m_{1} \in E\left(\Gamma_{1}\right)}\left[\frac{\operatorname{deg}_{\Gamma_{1}}\left(l_{1}\right) \cdot \operatorname{deg}_{\Gamma_{1}}\left(m_{1}\right)}{\operatorname{deg}_{\Gamma_{1}}\left(l_{1}\right)+\operatorname{deg}_{\Gamma_{1}}\left(m_{1}\right)-2}\right]^{\left.3\left[\left(\operatorname{deg}_{\Gamma_{1}}\left(l_{1}\right) \cdot \operatorname{deg}_{\Gamma_{1}}\left(m_{1}\right)\right) /\left(\operatorname{deg}_{\Gamma_{1}}\left(l_{1}\right)+\operatorname{deg}_{\Gamma_{1}}\left(m_{1}\right)-2\right)\right]^{3}\right]}\right. \\
& =\log \left(A\left(\Gamma_{1}\right)\right)-\frac{1}{A\left(\Gamma_{1}\right)} \log \left[\begin{array}{c}
\prod_{l_{1} m_{1} \in E_{\{2,2\}}}\left[\frac{\operatorname{deg}_{\Gamma_{1}}\left(l_{1}\right) \cdot \operatorname{deg}_{\Gamma_{1}}\left(m_{1}\right)}{\operatorname{deg}_{\Gamma_{1}}\left(l_{1}\right)+\operatorname{deg}_{\Gamma_{1}}\left(m_{1}\right)-2}\right]^{3\left[\left(\operatorname{deg}_{\Gamma_{1}}\left(l_{1}\right) \cdot \operatorname{deg}_{\Gamma_{1}}\left(m_{1}\right)\right) /\left(\operatorname{deg}_{\Gamma_{1}}\left(l_{1}\right)+\operatorname{deg}_{\Gamma_{1}}\left(m_{1}\right)-2\right)\right]^{3}} \\
\times \prod_{l_{1} m_{1} \in E_{\{2,3\}}}\left[\frac{\operatorname{deg}_{\Gamma_{1}}\left(l_{1}\right) \cdot \operatorname{deg}_{\Gamma_{1}}\left(m_{1}\right)}{\operatorname{deg}_{\Gamma_{1}}\left(l_{1}\right)+\operatorname{deg}_{\Gamma_{1}}\left(m_{1}\right)-2}\right]^{3\left[\left(\operatorname{deg}_{\Gamma_{1}}\left(l_{1}\right) \cdot \operatorname{deg}_{\Gamma_{1}}\left(m_{1}\right)\right) /\left(\operatorname{deg}_{\Gamma_{1}}\left(l_{1}\right)+\operatorname{deg}_{\Gamma_{1}}\left(m_{1}\right)-2\right)\right]^{3}} \\
\left.\times \prod_{l_{1} m_{1} \in E_{\{3,3\}}}\left[\frac{\operatorname{deg}_{\Gamma_{1}}\left(l_{1}\right) \cdot \operatorname{deg}_{\Gamma_{1}}\left(m_{1}\right)}{\operatorname{deg}_{\Gamma_{1}}\left(l_{1}\right)+\operatorname{deg}_{\Gamma_{1}}\left(m_{1}\right)-2}\right]^{3\left[\left(\operatorname{deg}_{\Gamma_{1}}\left(l_{1}\right) \cdot \operatorname{deg}_{\Gamma_{1}}\left(m_{1}\right)\right) /\left(\operatorname{deg}_{\Gamma_{1}}\left(l_{1}\right)+\operatorname{deg}_{\Gamma_{1}}\left(m_{1}\right)-2\right)\right]^{3}}\right]
\end{array}\right. \\
& =\log \left(\frac{1}{32}(14892 s-1675) r+128 s\right)-\frac{1}{[(1 / 32)(14892 s-1675) r+128 s]} \\
& \log \left[\left[[4 s+2 r] \times[8]^{[8]}\right] \times\left[[24 r s+12 s] \times[8]^{[8]}\right] \times\left[[24 r s-6 r] \times\left[\frac{729}{64}\right]^{[729 / 64]}\right]\right] .
\end{aligned}
$$

3.8. Atom Bond Connectivity Entropy. By using the edge partition in Table 3,

$$
A B C\left(\Gamma_{1}\right)=((12 s+1) r+8 s) \sqrt{2}+(16 s-4) r .
$$

Then, using the equation of atom bond connectivity entropy, the result is obtained as

$$
\operatorname{ENT}_{A B C}\left(\Gamma_{1}\right)=\log \left(A B C\left(\Gamma_{1}\right)\right)-\frac{1}{A B C\left(\Gamma_{1}\right)} \log \left[\prod_{l_{1} m_{1} \in E\left(\Gamma_{1}\right)}\left[\sqrt{\frac{\operatorname{deg}_{\Gamma_{1}}\left(l_{1}\right)+\operatorname{deg}_{\Gamma_{1}}\left(m_{1}\right)-2}{\operatorname{deg}_{\Gamma_{1}}\left(l_{1}\right) \cdot \operatorname{deg}_{\Gamma_{1}}\left(m_{1}\right)}}\right]^{\left[\sqrt{\left(\operatorname{deg}_{\Gamma_{1}}\left(l_{1}\right)+\operatorname{deg}_{\Gamma_{1}}\left(m_{1}\right)-2\right) /\left(\operatorname{deg}_{\Gamma_{1}}\left(l_{1}\right) \cdot \operatorname{deg}_{\Gamma_{1}}\left(m_{1}\right)\right)}\right]}\right.
$$




$$
\begin{gathered}
{\left[\prod_{l_{1} m_{1} \in E_{\{2,2\}}}\left[\sqrt{\frac{\operatorname{deg}_{\Gamma_{1}}\left(l_{1}\right)+\operatorname{deg}_{\Gamma_{1}}\left(m_{1}\right)-2}{\operatorname{deg}_{\Gamma_{1}}\left(l_{1}\right) \cdot \operatorname{deg}_{\Gamma_{1}}\left(m_{1}\right)}}\right]\left[\sqrt{\left(\operatorname{deg}_{\Gamma_{1}}\left(l_{1}\right)+\operatorname{deg}_{\Gamma_{1}}\left(m_{1}\right)-2\right) /\left(\operatorname{deg}_{\Gamma_{1}}\left(l_{1}\right) \cdot \operatorname{deg}_{\Gamma_{1}}\left(m_{1}\right)\right)}\right]\right.} \\
\left.=\log \left(A B C\left(\Gamma_{1}\right)\right)-\frac{1}{A B C\left(\Gamma_{1}\right)} \log \left[\times \prod_{l_{1} m_{1} \in E_{\{2,3\}}}\left[\sqrt{\frac{\operatorname{deg}_{\Gamma_{1}}\left(l_{1}\right)+\operatorname{deg}_{\Gamma_{1}}\left(m_{1}\right)-2}{\operatorname{deg}_{\Gamma_{1}}\left(l_{1}\right) \cdot \operatorname{deg}_{\Gamma_{1}}\left(m_{1}\right)}}\right]^{\left[\sqrt{\left(\operatorname{deg}_{\Gamma_{1}}\left(l_{1}\right)+\operatorname{deg}_{\Gamma_{1}}\left(m_{1}\right)-2\right) /\left(\operatorname{deg}_{\Gamma_{1}}\left(l_{1}\right) \cdot \operatorname{deg}_{\Gamma_{1}}\left(m_{1}\right)\right)}\right]}\right] \times \prod_{l_{1} m_{1} \in E_{\{\{, 3\}}}\left[\sqrt{\frac{\operatorname{deg}_{\Gamma_{1}}\left(l_{1}\right)+\operatorname{deg}_{\Gamma_{1}}\left(m_{1}\right)-2}{\operatorname{deg}_{\Gamma_{1}}\left(l_{1}\right) \cdot \operatorname{deg}_{\Gamma_{1}}\left(m_{1}\right)}}\right]^{\left[\sqrt{\left(\operatorname{deg}_{\Gamma_{1}}\left(l_{1}\right)+\operatorname{deg}_{\Gamma_{1}}\left(m_{1}\right)-2\right) /\left(\operatorname{deg}_{\Gamma_{1}}\left(l_{1}\right) \cdot \operatorname{deg}_{\Gamma_{1}}\left(m_{1}\right)\right)}\right]}\right] \\
=\log (((12 s+1) r+8 s) \sqrt{2}+(16 s-4) r)-\frac{1}{[((12 s+1) r+8 s) \sqrt{2}+(16 s-4) r]} \\
\log \left[\left[[4 s+2 r] \times\left[\sqrt{\frac{1}{2}}\right]^{[\sqrt{1 / 2}]}\right] \times\left[[24 r s+12 s] \times\left[\sqrt{\frac{1}{2}}\right]^{[\sqrt{1 / 2}]}\right] \times\left[[24 r s-6 r] \times\left[\frac{2}{3}\right]^{[2 / 3]}\right]\right] .
\end{gathered}
$$

3.9. Geometric Arithmetic Entropy. By using the edge partition in Table 3,

$$
\mathrm{GA}\left(\Gamma_{1}\right)=4 s-4 r+\left(\frac{48}{5}\right) \sqrt{6} r s+\left(\frac{24}{5}\right) \sqrt{6} s+24 r s .
$$

$$
\begin{aligned}
& \mathrm{ENT}_{\mathrm{GA}}\left(\Gamma_{1}\right)=\log \left(\mathrm{GA}\left(\Gamma_{1}\right)\right)-\frac{1}{\mathrm{GA}\left(\Gamma_{1}\right)} \log \left[\prod_{l_{1} m_{1} \in E\left(\Gamma_{1}\right)}\left[\frac{2 \sqrt{\operatorname{deg}_{\Gamma_{1}}\left(l_{1}\right) \cdot \operatorname{deg}_{\Gamma_{1}}\left(m_{1}\right)}}{\operatorname{deg}_{\Gamma_{1}}\left(l_{1}\right)+\operatorname{deg}_{\Gamma_{1}}\left(m_{1}\right)}\right]^{\left[2 \sqrt{\operatorname{deg}_{\Gamma_{1}}\left(l_{1}\right) \cdot \operatorname{deg}_{\Gamma_{1}}\left(m_{1}\right)} / \operatorname{deg}_{\Gamma_{1}}\left(l_{1}\right)+\operatorname{deg}_{\Gamma_{1}}\left(m_{1}\right)\right]}\right]
\end{aligned}
$$

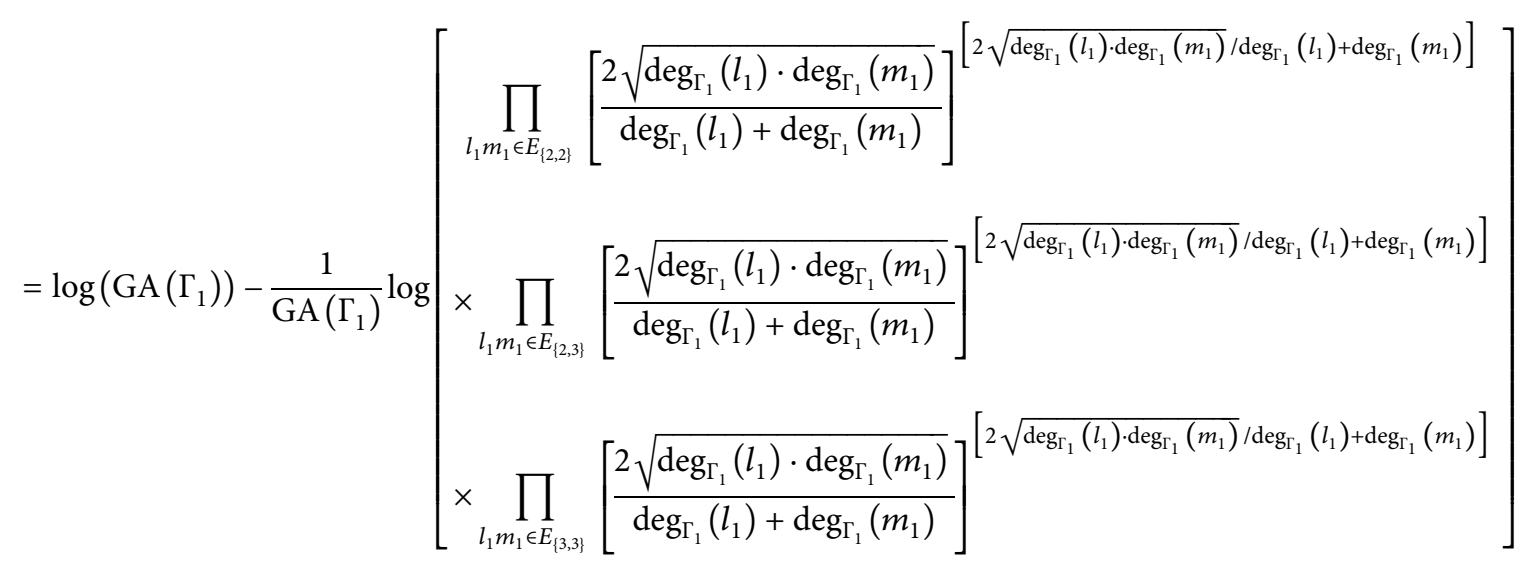

$$
\begin{aligned}
& =\log \left(4 s-4 r+\left(\frac{48}{5}\right) \sqrt{6} r s+\left(\frac{24}{5}\right) \sqrt{6} s+24 r s\right)-\frac{1}{[4 s-4 r+(48 / 5) \sqrt{6} r s+(24 / 5) \sqrt{6} s+24 r s]} \\
& \log \left[\left[[4 s+2 r] \times[1]^{[1]}\right] \times\left[[24 r s+12 s] \times\left[\frac{2 \sqrt{6}}{5}\right]^{[2 \sqrt{6} / 5]}\right] \times\left[[24 r s-6 r] \times[1]^{[1]}\right]\right] .
\end{aligned}
$$


3.10. Symmetric Division Deg (SDD) Entropy. By using the edge partition in Table 3,

$$
\operatorname{SDD}\left(\Gamma_{1}\right)=100 r s-8 r+34 s .
$$

Then, using the equation of Symmetric division deg entropy, the result is obtained as

$$
\begin{aligned}
& \operatorname{ENT}_{\mathrm{SDD}}\left(\Gamma_{1}\right)=\log \left(\operatorname{SDD}\left(\Gamma_{1}\right)\right)-\frac{1}{\operatorname{SDD}\left(\Gamma_{1}\right)} \log \left[\prod_{l_{1} m_{1} \in E\left(\Gamma_{1}\right)}\left[\frac{\operatorname{deg}_{\Gamma_{1}}\left(l_{1}\right)}{\operatorname{deg}_{\Gamma_{1}}\left(m_{1}\right)}+\frac{\operatorname{deg}_{\Gamma_{1}}\left(m_{1}\right)}{\operatorname{deg}_{\Gamma_{1}}\left(l_{1}\right)}\right]^{\left[\left(\operatorname{deg}_{\Gamma_{1}}\left(l_{1}\right) / \operatorname{deg}_{\Gamma_{1}}\left(m_{1}\right)\right)+\left(\operatorname{deg}_{\Gamma_{1}}\left(m_{1}\right) / \operatorname{deg}_{\Gamma_{1}}\left(l_{1}\right)\right)\right]}\right] \\
& =\log \left(\operatorname{SDD}\left(\Gamma_{1}\right)\right)-\frac{1}{\operatorname{SDD}\left(\Gamma_{1}\right)} \log \left[\begin{array}{c}
\left.\prod_{l_{1} m_{1} \in E_{\{2,2\}}}\left[\frac{\operatorname{deg}_{\Gamma_{1}}\left(l_{1}\right)}{\operatorname{deg}_{\Gamma_{1}}\left(m_{1}\right)}+\frac{\operatorname{deg}_{\Gamma_{1}}\left(m_{1}\right)}{\operatorname{deg}_{\Gamma_{1}}\left(l_{1}\right)}\right]^{\left[\left(\operatorname{deg}_{\Gamma_{1}}\left(l_{1}\right) / \operatorname{deg}_{\Gamma_{1}}\left(m_{1}\right)\right)+\left(\operatorname{deg}_{\Gamma_{1}}\left(m_{1}\right) / \operatorname{deg}_{\Gamma_{1}}\left(l_{1}\right)\right)\right]}\right] \\
\left.\times \prod_{l_{1} m_{1} \in E_{\{2,3\}}}\left[\frac{\operatorname{deg}_{\Gamma_{1}}\left(l_{1}\right)}{\operatorname{deg}_{\Gamma_{1}}\left(m_{1}\right)}+\frac{\operatorname{deg}_{\Gamma_{1}}\left(m_{1}\right)}{\operatorname{deg}_{\Gamma_{1}}\left(l_{1}\right)}\right]^{\left[\left(\operatorname{deg}_{\Gamma_{1}}\left(l_{1}\right) / \operatorname{deg}_{\Gamma_{1}}\left(m_{1}\right)\right)+\left(\operatorname{deg}_{\Gamma_{1}}\left(m_{1}\right) / \operatorname{deg}_{\Gamma_{1}}\left(l_{1}\right)\right)\right]}\right] \\
\left.\times \prod_{l_{1} m_{1} \in E_{\{3,3\}}}\left[\frac{\operatorname{deg}_{\Gamma_{1}}\left(l_{1}\right)}{\operatorname{deg}_{\Gamma_{1}}\left(m_{1}\right)}+\frac{\operatorname{deg}_{\Gamma_{1}}\left(m_{1}\right)}{\operatorname{deg}_{\Gamma_{1}}\left(l_{1}\right)}\right]^{\left[\left(\operatorname{deg}_{\Gamma_{1}}\left(l_{1}\right) / \operatorname{deg}_{\Gamma_{1}}\left(m_{1}\right)\right)+\left(\operatorname{deg}_{\Gamma_{1}}\left(m_{1}\right) / \operatorname{deg}_{\Gamma_{1}}\left(l_{1}\right)\right)\right]}\right]
\end{array}\right. \\
& =\log (100 r s-8 r+34 s)-\frac{1}{[100 r s-8 r+34 s]} \\
& \log \left[\left[[4 s+2 r] \times[2]^{[2]}\right] \times\left[[24 r s+12 s] \times\left[\frac{13}{6}\right]^{[13 / 6]}\right] \times\left[[24 r s-6 r] \times[2]^{[2]}\right]\right] \text {. }
\end{aligned}
$$

3.11. Randić Entropy. By using the edge partition in Table 3,

$$
R\left(\Gamma_{1}\right)=4 \sqrt{6} r s+2 \sqrt{6} s+8 r s-r+2 s .
$$

Then, using the equation of Randic entropy, the result is obtained as

$$
\begin{aligned}
\operatorname{ENT}_{R}\left(\Gamma_{1}\right) & =\log \left(R\left(\Gamma_{1}\right)\right)-\frac{1}{R\left(\Gamma_{1}\right)} \log \left[\prod_{l_{1} m_{1} \in E\left(\Gamma_{1}\right)}\left[\frac{1}{\sqrt{\operatorname{deg}_{\Gamma_{1}}\left(l_{1}\right) \cdot \operatorname{deg}_{\Gamma_{1}}\left(m_{1}\right)}}\right]^{\left[\left(1 / \sqrt{\operatorname{deg}_{\Gamma_{1}}\left(l_{1}\right) \cdot \operatorname{deg}_{\Gamma_{1}}\left(m_{1}\right)}\right)\right]}\right] \\
& =\log \left(R\left(\Gamma_{1}\right)\right)-\frac{1}{R\left(\Gamma_{1}\right)} \log \left[\begin{array}{l}
\prod_{l_{1} m_{1} \in E_{\{2,2\}}}\left[\frac{1}{\sqrt{\operatorname{deg}_{\Gamma_{1}}\left(l_{1}\right) \cdot \operatorname{deg}_{\Gamma_{1}}\left(m_{1}\right)}}\right]^{\left[\left(1 / \sqrt{\operatorname{deg}_{\Gamma_{1}}\left(l_{1}\right) \cdot \operatorname{deg}_{\Gamma_{1}}\left(m_{1}\right)}\right)\right]} \\
\times \prod_{l_{1} m_{1} \in E_{\{2,3\}}}\left[\frac{1}{\sqrt{\operatorname{deg}_{\Gamma_{1}}\left(l_{1}\right) \cdot \operatorname{deg}_{\Gamma_{1}}\left(m_{1}\right)}}\right]^{\left[\left(1 / \sqrt{\operatorname{deg}_{\Gamma_{1}}\left(l_{1}\right) \cdot \operatorname{deg}_{\Gamma_{1}}\left(m_{1}\right)}\right)\right]} \\
\times \prod_{l_{1} m_{1} \in E_{\{3,3\}}}\left[\frac{1}{\sqrt{\operatorname{deg}_{\Gamma_{1}}\left(l_{1}\right) \cdot \operatorname{deg}_{\Gamma_{1}}\left(m_{1}\right)}}\right]^{\left[\left(1 / \sqrt{\operatorname{deg}_{\Gamma_{1}}\left(l_{1}\right) \cdot \operatorname{deg}_{\Gamma_{1}}\left(m_{1}\right)}\right)\right]}
\end{array}\right]
\end{aligned}
$$




$$
\begin{aligned}
= & \log (4 \sqrt{6} r s+2 \sqrt{6} s+8 r s-r+2 s)-\frac{1}{[4 \sqrt{6} r s+2 \sqrt{6} s+8 r s-r+2 s]} \\
& \log \left[\left[[4 s+2 r] \times\left[\frac{1}{2}\right]^{[1 / 2]}\right] \times\left[[24 r s+12 s] \times\left[\frac{1}{\sqrt{6}}\right]^{[1 / \sqrt{6}]}\right] \times\left[[24 r s-6 r] \times\left[\frac{1}{3}\right]^{[1 / 3]}\right]\right] .
\end{aligned}
$$

3.12. Forgotten Entropy. By using the edge partition in Table 3,

$$
F\left(\Gamma_{1}\right)=744 r s-92 r+188 s .
$$

$$
\begin{aligned}
\operatorname{ENT}_{F}\left(\Gamma_{1}\right)= & \log \left(F\left(\Gamma_{1}\right)\right)-\frac{1}{F\left(\Gamma_{1}\right)} \log \left[\prod_{l_{1} m_{1} \in E\left(\Gamma_{1}\right)}\left[\operatorname{deg}_{\Gamma_{1}}\left(l_{1}\right)^{2}+\operatorname{deg}_{\Gamma_{1}}\left(m_{1}\right)^{2}\right]^{\left[\operatorname{deg}_{\Gamma_{1}}\left(l_{1}\right)^{2}+\operatorname{deg}_{\Gamma_{1}}\left(m_{1}\right)^{2}\right]}\right] \\
= & \left.\log \left(F\left(\Gamma_{1}\right)\right)-\frac{1}{F\left(\Gamma_{1}\right)} \log \left[\times \prod_{l_{1} m_{1} \in E_{\{2,2\}}}\left[\operatorname{deg}_{\Gamma_{1}}\left(l_{1}\right)^{2}+\operatorname{deg}_{\Gamma_{1}}\left(m_{1}\right)^{2}\right]^{\left[\operatorname{deg}_{\Gamma_{1}}\left(l_{1}\right)^{2}+\operatorname{deg}_{\Gamma_{1}}\left(m_{1}\right)^{2}\right]}\right] \times \prod_{l_{12,3\}}}\left[\operatorname{deg}_{\Gamma_{1}}\left(l_{1}\right)^{2}+\operatorname{deg}_{\Gamma_{1}}\left(m_{1}\right)^{2}\right]^{\left[\operatorname{deg}_{\Gamma_{1}}\left(l_{1}\right)^{2}+\operatorname{deg}_{\Gamma_{1}}\left(m_{1}\right)^{2}\right]}\right] \\
= & \log (744 r s-92 r+188 s)-\frac{1}{[744 r s-92 r+188 s]} \\
& \log \left[\left[[4 s+2 r] \times[8]^{[8]}\right] \times\left[[24 r s+12 s] \times[13]^{[13]}\right] \times\left[[24 r s-6 r] \times[18]^{[18]}\right]\right] .
\end{aligned}
$$

3.13. Sum-Connectivity Entropy. By using the edge partition in Table 3,

$$
\chi\left(\Gamma_{1}\right)=2 s+r+\left(\frac{24}{5}\right) \sqrt{5} r s+\left(\frac{12}{5}\right) \sqrt{5} s+4 \sqrt{6} r s-\sqrt{6} r .
$$

Then, using the equation of sum-connectivity entropy, the result is obtained as

$$
\operatorname{ENT}_{\chi}\left(\Gamma_{1}\right)=\log \left(\chi\left(\Gamma_{1}\right)\right)-\frac{1}{\chi\left(\Gamma_{1}\right)} \log \left[\prod_{l_{1} m_{1} \in E\left(\Gamma_{1}\right)}\left[\frac{1}{\sqrt{\operatorname{deg}_{\Gamma_{1}}\left(l_{1}\right)+\operatorname{deg}_{\Gamma_{1}}\left(m_{1}\right)}}\right]^{\left[\left(1 / \sqrt{\operatorname{deg}_{\Gamma_{1}}\left(l_{1}\right)+\operatorname{deg}_{\Gamma_{1}}\left(m_{1}\right)}\right)\right]}\right]
$$




$$
\begin{aligned}
& =\log \left(F\left(\Gamma_{1}\right)\right)-\frac{1}{F\left(\Gamma_{1}\right)} \log \left[\begin{array}{c}
\left.\prod_{l_{1} m_{1} \in E_{\{2,2\}}}\left[\frac{1}{\sqrt{\operatorname{deg}_{\Gamma_{1}}\left(l_{1}\right)+\operatorname{deg}_{\Gamma_{1}}\left(m_{1}\right)}}\right]^{\left[\left(1 / \sqrt{\operatorname{deg}_{\Gamma_{1}}\left(l_{1}\right)+\operatorname{deg}_{\Gamma_{1}}\left(m_{1}\right)}\right)\right]}\right] \\
\left.\times \prod_{l_{1} m_{1} \in E_{\{2,3\}}}\left[\frac{1}{\sqrt{\operatorname{deg}_{\Gamma_{1}}\left(l_{1}\right)+\operatorname{deg}_{\Gamma_{1}}\left(m_{1}\right)}}\right]^{\left[\left(1 / \sqrt{\operatorname{deg}_{\Gamma_{1}}\left(l_{1}\right)+\operatorname{deg}_{\Gamma_{1}}\left(m_{1}\right)}\right)\right]}\right] \\
\left.\times \prod_{l_{1} m_{1} \in E_{\{3,3\}}}\left[\frac{1}{\sqrt{\operatorname{deg}_{\Gamma_{1}}\left(l_{1}\right)+\operatorname{deg}_{\Gamma_{1}}\left(m_{1}\right)}}\right]^{\left[\left(1 / \sqrt{\operatorname{deg}_{\Gamma_{1}}\left(l_{1}\right)+\operatorname{deg}_{\Gamma_{1}}\left(m_{1}\right)}\right)\right]}\right]
\end{array}\right. \\
& =\log \left(2 s+r+\left(\frac{24}{5}\right) \sqrt{5} r s+\left(\frac{12}{5}\right) \sqrt{5} s+4 \sqrt{6} r s-\sqrt{6} r\right)-\frac{1}{[2 s+r+(24 / 5) \sqrt{5} r s+(12 / 5) \sqrt{5} s+4 \sqrt{6} r s-\sqrt{6} r]} \\
& \log \left[\left[[4 s+2 r] \times\left[\frac{1}{2}\right]^{[1 / 2]}\right] \times\left[[24 r s+12 s] \times\left[\frac{1}{\sqrt{5}}\right]^{[1 / \sqrt{5}]}\right] \times\left[[24 r s-6 r] \times\left[\frac{1}{\sqrt{6}}\right]^{[1 / \sqrt{6}]}\right]\right]
\end{aligned}
$$

3.14. First Redefined Zagreb Entropy. By using the edge partition in Table 3,

$$
\operatorname{ReZG}_{1}\left(\Gamma_{1}\right)=36 r s-2 r+14 s .
$$

$$
\begin{aligned}
& \operatorname{ENT}_{\mathrm{ReZG}_{1}}\left(\Gamma_{1}\right)=\log \left(\operatorname{ReZG}_{1}\left(\Gamma_{1}\right)\right)-\frac{1}{\operatorname{ReZG}_{1}\left(\Gamma_{1}\right)} \log \left[\prod_{l_{1} m_{1} \in E\left(\Gamma_{1}\right)}\left[\frac{\operatorname{deg}_{\Gamma_{1}}\left(l_{1}\right)+\operatorname{deg}_{\Gamma_{1}}\left(m_{1}\right)}{\operatorname{deg}_{\Gamma_{1}}\left(l_{1}\right) \cdot \operatorname{deg}_{\Gamma_{1}}\left(m_{1}\right)}\right]^{\left[\left(\operatorname{deg}_{\Gamma_{1}}\left(l_{1}\right)+\operatorname{deg}_{\Gamma_{1}}\left(m_{1}\right)\right) /\left(\operatorname{deg}_{\Gamma_{1}}\left(l_{1}\right) \cdot \operatorname{deg}_{\Gamma_{1}}\left(m_{1}\right)\right)\right]}\right] \\
& =\log \left(\operatorname{ReZG}_{1}\left(\Gamma_{1}\right)\right)-\frac{1}{\operatorname{ReZG}_{1}\left(\Gamma_{1}\right)} \log \left[\begin{array}{c}
\left.\prod_{l_{1} m_{1} \in E_{\{2,2\}}}\left[\frac{\operatorname{deg}_{\Gamma_{1}}\left(l_{1}\right)+\operatorname{deg}_{\Gamma_{1}}\left(m_{1}\right)}{\operatorname{deg}_{\Gamma_{1}}\left(l_{1}\right) \cdot \operatorname{deg}_{\Gamma_{1}}\left(m_{1}\right)}\right]^{\left[\left(\operatorname{deg}_{\Gamma_{1}}\left(l_{1}\right)+\operatorname{deg}_{\Gamma_{1}}\left(m_{1}\right)\right) /\left(\operatorname{deg}_{\Gamma_{1}}\left(l_{1}\right) \cdot \operatorname{deg}_{\Gamma_{1}}\left(m_{1}\right)\right)\right]}\right] \\
\left.\times \prod_{l_{1} m_{1} \in E_{\{2,3\}}}\left[\frac{\operatorname{deg}_{\Gamma_{1}}\left(l_{1}\right)+\operatorname{deg}_{\Gamma_{1}}\left(m_{1}\right)}{\operatorname{deg}_{\Gamma_{1}}\left(l_{1}\right) \cdot \operatorname{deg}_{\Gamma_{1}}\left(m_{1}\right)}\right]^{\left[\left(\operatorname{deg}_{\Gamma_{1}}\left(l_{1}\right)+\operatorname{deg}_{\Gamma_{1}}\left(m_{1}\right)\right) /\left(\operatorname{deg}_{\Gamma_{1}}\left(l_{1}\right) \cdot \operatorname{deg}_{\Gamma_{1}}\left(m_{1}\right)\right)\right]}\right] \\
\left.\times \prod_{l_{1} m_{1} \in E_{\{3,3\}}}\left[\frac{\operatorname{deg}_{\Gamma_{1}}\left(l_{1}\right)+\operatorname{deg}_{\Gamma_{1}}\left(m_{1}\right)}{\operatorname{deg}_{\Gamma_{1}}\left(l_{1}\right) \cdot \operatorname{deg}_{\Gamma_{1}}\left(m_{1}\right)}\right]^{\left[\left(\operatorname{deg}_{\Gamma_{1}}\left(l_{1}\right)+\operatorname{deg}_{\Gamma_{1}}\left(m_{1}\right)\right) /\left(\operatorname{deg}_{\Gamma_{1}}\left(l_{1}\right) \cdot \operatorname{deg}_{\Gamma_{1}}\left(m_{1}\right)\right)\right]}\right]
\end{array}\right. \\
& =\log (36 r s-2 r+14 s)-\frac{1}{[36 r s-2 r+14 s]} \\
& \log \left[\left[[4 s+2 r] \times[1]^{[1]}\right] \times\left[[24 r s+12 s] \times\left[\frac{5}{6}\right]^{[5 / 6]}\right] \times\left[[24 r s-6 r] \times\left[\frac{2}{3}\right]^{[2 / 3]}\right]\right]
\end{aligned}
$$

3.15. Second Redefined Zagreb Entropy. By using the edge partition in Table 3,

$$
\operatorname{ReZG}_{1}\left(\Gamma_{1}\right)=\left(\frac{92}{5}\right) s-7 r+\left(\frac{324}{5}\right) r s .
$$


Then, using the equation of the second redefined Zagreb entropy, the result is obtained as

$$
\begin{aligned}
& \operatorname{ENT}_{\mathrm{ReZG}_{2}}\left(\Gamma_{1}\right)=\log \left(\operatorname{ReZG}_{2}\left(\Gamma_{1}\right)\right)-\frac{1}{\operatorname{ReZG}_{2}\left(\Gamma_{1}\right)} \log \left[\prod_{l_{1} m_{1} \in E\left(\Gamma_{1}\right)}\left[\frac{\operatorname{deg}_{\Gamma_{1}}\left(l_{1}\right) \cdot \operatorname{deg}_{\Gamma_{1}}\left(m_{1}\right)}{\operatorname{deg}_{\Gamma_{1}}\left(l_{1}\right)+\operatorname{deg}_{\Gamma_{1}}\left(m_{1}\right)}\right]^{\left[\left(\operatorname{deg}_{\Gamma_{1}}\left(l_{1}\right) \cdot \operatorname{deg}_{\Gamma_{1}}\left(m_{1}\right)\right) /\left(\operatorname{deg}_{\Gamma_{1}}\left(l_{1}\right)+\operatorname{deg}_{\Gamma_{1}}\left(m_{1}\right)\right)\right]}\right] \\
& =\log \left(\operatorname{ReZG}_{2}\left(\Gamma_{1}\right)\right)-\frac{1}{\operatorname{ReZG}_{2}\left(\Gamma_{1}\right)} \log \left[\begin{array}{l}
\left.\prod_{l_{1} m_{1} \in E_{\{2,2\}}}\left[\frac{\operatorname{deg}_{\Gamma_{1}}\left(l_{1}\right) \cdot \operatorname{deg}_{\Gamma_{1}}\left(m_{1}\right)}{\operatorname{deg}_{\Gamma_{1}}\left(l_{1}\right)+\operatorname{deg}_{\Gamma_{1}}\left(m_{1}\right)}\right]^{\left[\left(\operatorname{deg}_{\Gamma_{1}}\left(l_{1}\right) \cdot \operatorname{deg}_{\Gamma_{1}}\left(m_{1}\right)\right) /\left(\operatorname{deg}_{\Gamma_{1}}\left(l_{1}\right)+\operatorname{deg}_{\Gamma_{1}}\left(m_{1}\right)\right)\right]}\right] \\
\left.\times \prod_{l_{1} m_{1} \in E_{\{2,3\}}}\left[\frac{\operatorname{deg}_{\Gamma_{1}}\left(l_{1}\right) \cdot \operatorname{deg}_{\Gamma_{1}}\left(m_{1}\right)}{\operatorname{deg}_{\Gamma_{1}}\left(l_{1}\right)+\operatorname{deg}_{\Gamma_{1}}\left(m_{1}\right)}\right]^{\left[\left(\operatorname{deg}_{\Gamma_{1}}\left(l_{1}\right) \cdot \operatorname{deg}_{\Gamma_{1}}\left(m_{1}\right)\right) /\left(\operatorname{deg}_{\Gamma_{1}}\left(l_{1}\right)+\operatorname{deg}_{\Gamma_{1}}\left(m_{1}\right)\right)\right]}\right] \\
\left.\times \prod_{l_{1} m_{1} \in E_{\{3,3\}}}\left[\frac{\operatorname{deg}_{\Gamma_{1}}\left(l_{1}\right) \cdot \operatorname{deg}_{\Gamma_{1}}\left(m_{1}\right)}{\operatorname{deg}_{\Gamma_{1}}\left(l_{1}\right)+\operatorname{deg}_{\Gamma_{1}}\left(m_{1}\right)}\right]^{\left[\left(\operatorname{deg}_{\Gamma_{1}}\left(l_{1}\right) \cdot \operatorname{deg}_{\Gamma_{1}}\left(m_{1}\right)\right) /\left(\operatorname{deg}_{\Gamma_{1}}\left(l_{1}\right)+\operatorname{deg}_{\Gamma_{1}}\left(m_{1}\right)\right)\right]}\right]
\end{array}\right. \\
& =\log \left(\left(\frac{92}{5}\right) s-7 r+\left(\frac{324}{5}\right) r s\right)-\frac{1}{[(92 / 5) s-7 r+(324 / 5) r s]} \\
& \log \left[\left[[4 s+2 r] \times[1]^{[1]}\right] \times\left[[24 r s+12 s] \times\left[\frac{6}{5}\right]^{[6 / 5]}\right] \times\left[[24 r s-6 r] \times\left[\frac{3}{2}\right]^{[3 / 2]}\right]\right] .
\end{aligned}
$$

Theorem 1. If $\Gamma_{1}$ is the $R K(r, s)$, then the irregularity-based descriptors are expressed as follows:

(1) $\operatorname{VAR}\left(\Gamma_{1}\right)=\left(2\left(36 r^{2} s^{2}+36 r s^{2}-r^{2}-4 r s+\right.\right.$

$\left.\left.5 s^{2}\right) /(18 r s-r+7 s)^{2}\right)$

(2) $\operatorname{IR} 1\left(\Gamma_{1}\right)=\left(4\left(180 r^{2} s^{2}+1434 r s^{2}-5 r^{2}-115 r s+\right.\right.$ $\left.\left.462 s^{2}\right) /(18 r s-r+7 s)\right)$

(3) $\operatorname{IR} 2\left(\Gamma_{1}\right)=$

$(1 / 2)(18 \sqrt{2} \sqrt{(180 r s-23 r+44 s) /(12 r s-r+4 s) r s}-$

$\sqrt{2} \sqrt{(180 r s-23 r+44 s) /(12 r s-r+4 s) r}+$

$7 \sqrt{2} \sqrt{(180 r s-23 r+44 s) /(12 r s-r+4 s) s}-96 r s+$ $8 r-32 s /(18 r s-r+7 s))$

(4) $\operatorname{IRDIF}\left(\Gamma_{1}\right)=(1 / 3)(24 r s+12 s)$

(5) $A L\left(\Gamma_{1}\right)=24 r s+12 s$

(6) $\operatorname{IRL}\left(\Gamma_{1}\right)=0.40545(24 r s+12 s)$

(7) $\operatorname{IRLU}\left(\Gamma_{1}\right)=(0.40545 / 2)(24 r s+12 s)$

(8) $\operatorname{IRLF}\left(\Gamma_{1}\right)=(1 / \sqrt{6})(24 r s+12 s)$

(9) $\operatorname{IRF}\left(\Gamma_{1}\right)=24 r s+12 s$

(10) $\operatorname{IRLA}\left(\Gamma_{1}\right)=(2 / 5)(24 r s+12 s)$

(11) $\operatorname{IRD} D_{1}\left(\Gamma_{1}\right)=0.69315(24 r s+12 s)$

(12) $\operatorname{IRA}\left(\Gamma_{1}\right)=0.016832(24 r s+12 s)$

(13) $\operatorname{IRB}\left(\Gamma_{1}\right)=0.10106(24 r s+12 s)$

(14) $\operatorname{IRB}\left(\Gamma_{1}\right)=(1 / 2)\left(\left(216 \sqrt{6} r^{2} s^{2}-12 \sqrt{6} r^{2} s+\right.\right.$ $192 \sqrt{6} r s^{2}-504 r^{2} s^{2}-6 \sqrt{6} r s+42 \sqrt{6} s^{2}+30 r^{2} s-$ $\left.\left.444 r s^{2}-r^{2}+11 r s-100 s^{2}\right) /(12 r s-r+4 s)\right)(18 r s-r+$ $7 s)$
(15) $\operatorname{IRGA}\left(\Gamma_{1}\right)=0.020391(24 r s+12 s)$

(16) $\operatorname{IRR}_{t}\left(\Gamma_{1}\right)=(1 / 2)(24 r s+12 s)$

\section{Numerical and Graphical Analysis of $\operatorname{RK}(r, s)$}

In this section, by changing the values of the variables $r$ and $s$ from 1 to 9 , the numerical values of VDB entropy measures are obtained using the analytical expressions of the rectangular Kekulene system. Tables 4 and 5 show the results of these calculations. By looking at their numerical values, the comparison can be done easily to see whether there are any similarities or differences between the individual topological indices. Figures 2 and 3 show this behaviour as three-dimensional graphical representations. The differences between each topological index for a specific structure can be seen in these $3 \mathrm{D}$ plots.

As it is evident from the above calculations, it is quite convenient to compute topological indices. Since topological indices correlate well with physiochemical properties, these can be directly used to predict the behaviour of compounds. Such a theoretical analysis based on topology significantly reduces the time and efforts required for analysis of compounds, when compared to intensive quantum chemical calculations or laborious experiments. But, not all properties of a compound can be mathematically associated to topological indices. Some features would still require practical experiments to be studied. The prediction accuracy of QSAR/QSPR methods which use TIs can also be comparatively less than that of 
TABLe 4: Comparison table of $\mathrm{RK}[r, s]$.

\begin{tabular}{lccccccc}
\hline $\mathrm{RK}[r, s]$ & $\mathrm{ENT}_{M_{1}}\left(\Gamma_{1}\right)$ & $\mathrm{ENT}_{M_{2}}\left(\Gamma_{1}\right)$ & $\mathrm{ENT}_{M_{2}^{m}}\left(\Gamma_{1}\right)$ & $\mathrm{ENT}_{R M_{2}}\left(\Gamma_{1}\right)$ & $\mathrm{ENT}_{\mathrm{HM}}\left(\Gamma_{1}\right)$ & $\mathrm{ENT}_{A}\left(\Gamma_{1}\right)$ & $\mathrm{ENT}_{A B C}\left(\Gamma_{1}\right)$ \\
\hline$[1,1]$ & 5.65 & 5.89 & 1.56 & 4.92 & 7.25 & 6.17 & 3.57 \\
{$[2,2]$} & 7.02 & 7.30 & 3.17 & 6.30 & 8.69 & 7.57 & 4.94 \\
{$[3,3]$} & 7.82 & 8.11 & 4.05 & 7.12 & 9.51 & 8.38 & 5.74 \\
{$[4,4]$} & 8.38 & 8.68 & 4.66 & 7.71 & 10.08 & 8.95 & 6.31 \\
{$[5,5]$} & 8.83 & 9.12 & 5.12 & 8.16 & 10.53 & 9.39 & 6.75 \\
\hline
\end{tabular}

TABle 5: Comparison table of $\mathrm{RK}[r, s]$.

\begin{tabular}{lccccccc}
\hline $\mathrm{RK}[r, s]$ & $\mathrm{ENT}_{\mathrm{GA}}\left(\Gamma_{1}\right)$ & $\mathrm{ENT}_{\mathrm{SDD}}\left(\Gamma_{1}\right)$ & $\mathrm{ENT}_{R}\left(\Gamma_{1}\right)$ & $\mathrm{ENT}_{F}\left(\Gamma_{1}\right)$ & $\mathrm{ENT}_{\chi}\left(\Gamma_{1}\right)$ & $\mathrm{ENT}_{\mathrm{ReZG}_{1}}\left(\Gamma_{1}\right)$ & $\mathrm{ENT}_{\mathrm{ReZG}_{2}}\left(\Gamma_{1}\right)$ \\
\hline$[1,1]$ & 3.96 & 4.84 & 2.89 & 6.60 & 3.03 & 3.72 & 4.24 \\
{$[2,2]$} & 5.31 & 6.08 & 4.30 & 8.03 & 4.44 & 5.06 & 5.85 \\
{$[3,3]$} & 6.11 & 6.87 & 5.11 & 8.84 & 5.25 & 6.60 & 6.40 \\
{$[4,4]$} & 6.68 & 7.43 & 5.69 & 9.41 & 5.83 & 6.97 \\
{$[5,5]$} & 7.12 & 7.87 & 6.13 & 9.85 & 6.27 & 6.85 & 7.42 \\
\hline
\end{tabular}

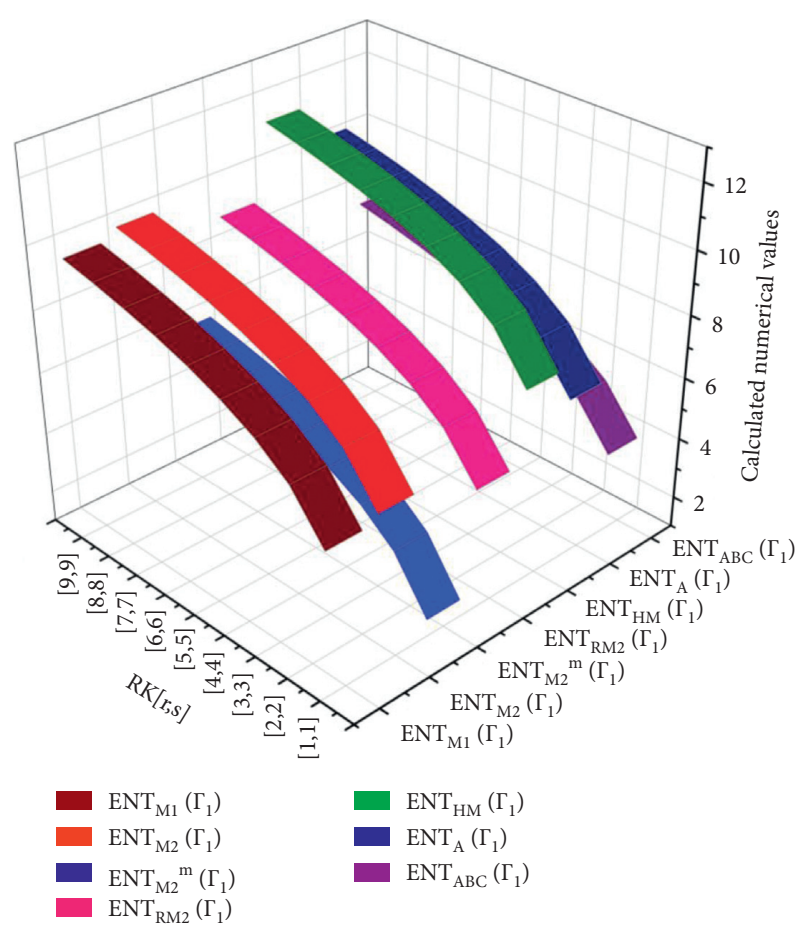

FIgURE 2: Graphical visualisation for Table 4.

experimental observations. However, TIs have been widely accepted by the scientific community as predictive methods for a subset of physiochemical properties due to their ease of use and universal applicability.

\section{Conclusion}

In this paper, the mathematical expressions of VDB entropy measures and irregularity-based indices have been computed for $\operatorname{RK}(r, s)$. Furthermore, the numerical values for these expressions of VDB entropy measures and irregularity-based indices are calculated using the

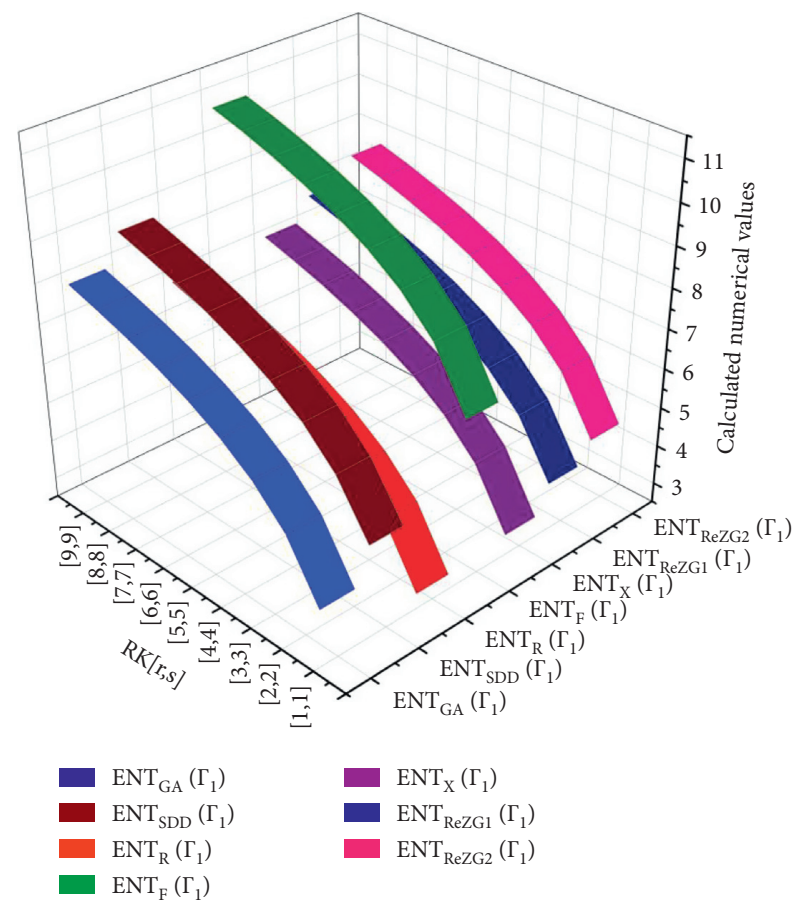

FIgURE 3: Graphical visualisation for Table 5.

obtained analytical expressions. The 3D graphical comparisons of these analytical expressions of VDB entropy measures have been represented visually to find the behavioural pattern of the molecular compound. The study of Wiener polarity-based TIs, eccentricity-based TIs, and the recent development of VDB indices for this structure can also be explored as they have never been studied earlier. It is still an area where further research could be done in the future.

\section{Data Availability}

No data were used to support this study. 


\section{Conflicts of Interest}

The authors declare that there are no conflicts of interest regarding the publication of this paper.

\section{Acknowledgments}

The authors would like to express their gratitude to the management of the Shiv Nadar Foundation for their continuous support and encouragement to do their research. This work was supported by the Science \& Technology Bureau of Chengdu under Grant 2020-YF09-00005-SN and the National Key Research and Development Program under Grant 2018YFB0904205.

\section{References}

[1] N. Trinajstić, Chemical Graph Theory, CRC Press, Boca Raton, FL, USA, 2nd edition, 1992.

[2] H. van de Waterbeemd, R. E. Carter, G. Grassy et al., "Glossary of terms used in computational drug design (IUPAC Recommendations 1997)," Pure and Applied Chemistry, vol. 69, no. 5, pp. 1137-1152, 1997.

[3] J. R. Dias, "Structure and electronic characteristics of coronoid polycyclic aromatic hydrocarbons as potential models of graphite layers with hole defects," The Journal of Physical Chemistry A, vol. 112, no. 47, pp. 12281-12292, 2008.

[4] H. Miyoshi, S. Nobusue, A. Shimizu, and Y. Tobe, "Nonalternant non-benzenoid kekulenes: the birth of a new kekulene family," Chemical Society Reviews, vol. 44, no. 18, pp. 6560-6577, 2015.

[5] J.-I. Aihara, "Magnetic resonance energy and topological resonance energy," Physical Chemistry Chemical Physics, vol. 18, no. 17, pp. 11847-11857, 2016.

[6] J.-I. Aihara, "Graph theory of ring-current diamagnetism," Bulletin of the Chemical Society of Japan, vol. 91, no. 2, pp. 274-303, 2018.

[7] U. Beser, M. Kastler, A. Maghsoumi et al., "A C216-nanographene molecule with defined cavity as extended coronoid," Journal of the American Chemical Society, vol. 138, no. 13, pp. 4322-4325, 2016.

[8] T. Parsons-Moss, S. Jones, J. Wang et al., "Reduction of plutonium in acidic solutions by mesoporous carbons," Journal of Radioanalytical and Nuclear Chemistry, vol. 307, no. 3, pp. 2593-2601, 2016.

[9] S. Shinkai, H. Koreishi, K. Ueda, T. Arimura, and O. Manabe, "Molecular design of calixarene-based uranophiles which exhibit remarkably high stability and selectivity," Journal of the American Chemical Society, vol. 109, no. 21, pp. 6371-6376, 1987.

[10] J. A. Shusterman, H. E. Mason, J. Bowers et al., "Development and testing of diglycolamide functionalized mesoporous silica for sorption of trivalent actinides and lanthanides," ACS Applied Materials \& Interfaces, vol. 7, no. 37, pp. 20591-20599, 2015.

[11] J. Cioslowski, "Additive nodal increments for approximate calculation of the total ?-electron energy of benzenoid hydrocarbons," Theoretica Chimica Acta, vol. 68, no. 4, pp. 315-319, 1985.

[12] J.-I. Aihara, M. Makino, T. Ishida, and J. R. Dias, "Analytical study of superaromaticity in Cycloarenes and related coronoid hydrocarbons," The Journal of Physical Chemistry A, vol. 117, no. 22, pp. 4688-4697, 2013.
[13] K. Julietraja and P. Venugopal, “Computation of degree-based topological descriptors using M-polynomial for coronoid systems," Polycyclic Aromatic Compounds, pp. 1-24, 2020.

[14] K. Julietraja, P. Venugopal, S. Prabhu, and J.-B. Liu, "Mpolynomial and degree-based molecular descriptors of certain classes of benzenoid systems," Polycyclic Aromatic Compounds, pp. 1-30, 2020.

[15] K. Julietraja, P. Venugopal, S. Prabhu, S. Deepa, and M. K. Siddiqui, "Molecular structural descriptors of donut benzenoid systems," Polycyclic Aromatic Compounds, pp. 127, 2021.

[16] K. Julietraja, P. Venugopal, S. Prabhu, A. K. Arulmozhi, and M. K. Siddiqui, "Structural analysis of three types of PAHs using entropy measures," Polycyclic Aromatic Compounds, pp. 1-31, 2021.

[17] D. B. West, An Introduction to Graph Theory, Prentice-Hall, Upper Saddle River, NJ, USA, 1996.

[18] C. E. Shannon, "A mathematical theory of communication," Bell System Technical Journal, vol. 27, no. 3, pp. 379-423, 1948.

[19] R. V. Solé and S. Valverde, "Information theory of complex networks: on evolution and architectural constraints," Complex Networks, vol. 650, pp. 189-207, 2004.

[20] Z. Chen, M. Dehmer, and Y. Shi, "A note on distance-based graph entropies," Entropy, vol. 16, no. 10, pp. 5416-5427, 2014.

[21] G. Chartrand, P. Erdös, and O. R. Oellermann, "How to define an irregular graph," The College Mathematics Journal, vol. 19, no. 1, pp. 36-42, 1988.

[22] Z. Majcher and J. Michael, "Highly irregular graphs with extreme numbers of edges," Discrete Mathematics, vol. 164, no. 1-3, pp. 237-242, 1997.

[23] F. K. Bell, "A note on the irregularity of graphs," Linear Algebra and Its Applications, vol. 161, pp. 45-54, 1992.

[24] T. Réti, R. Sharafdini, A. Dregelyi-Kiss, and H. Haghbin, "Graph irregularity indices used as molecular descriptors in QSPR studies," MATCH Communications in Mathematical and in Computer Chemistry, vol. 79, pp. 509-524, 2018.

[25] W. Gao, Z. Iqbal, Z. Iqbal, S. Akhter, M. Ishaq, and A. Aslam, "On irregularity descriptors of derived graphs," AIMS Mathematics, vol. 5, no. 5, pp. 4085-4107, 2020.

[26] A. Ghalavand and T. Sohail, "On some variations of the irregularity," Discrete Mathematics Letters, vol. 3, pp. 25-30, 2020.

[27] J. Zheng, S. Akhter, Z. Iqbal et al., "Irregularity measures of subdivision vertex-edge join of graphs," Journal of Chemistry, vol. 2021, Article ID 6673221, 12 pages, 2021.

[28] K. Julietraja, P. Venugopal, and P. Chellamani, "Topological analysis of PAHs using irregularity based indices," Biointerface research in applied chemistry, vol. 12, no. 3, pp. 29702987, 2021.

[29] K. Julietraja and P. Chellamani, "VDB analysis for zeolites LTA structures," Biointerface Research in Applied Chemistry, 2021. 